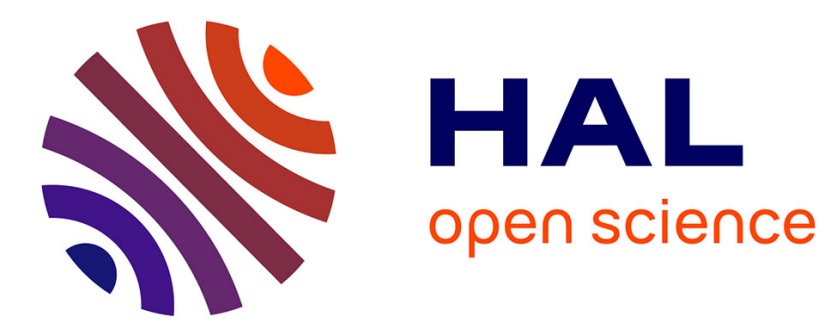

\title{
La répartition de l'ozone atmosphérique en fonction de l'altitude
}

Damien Barbier

\section{To cite this version:}

Damien Barbier. La répartition de l'ozone atmosphérique en fonction de l'altitude. J. Phys. Radium, 1934, 5 (6), pp.243-252. 10.1051/jphysrad:0193400506024300 . jpa-00233231

\section{HAL Id: jpa-00233231 https://hal.science/jpa-00233231}

Submitted on 1 Jan 1934

HAL is a multi-disciplinary open access archive for the deposit and dissemination of scientific research documents, whether they are published or not. The documents may come from teaching and research institutions in France or abroad, or from public or private research centers.
L'archive ouverte pluridisciplinaire HAL, est destinée au dépôt et à la diffusion de documents scientifiques de niveau recherche, publiés ou non, émanant des établissements d'enseignement et de recherche français ou étrangers, des laboratoires publics ou privés. 


\title{
LA RÉPARTITION DE L'OZONE ATMOSPHÉRIQUE EN FONGTION DE L'ALTITUDE
}

\author{
Par M. D. BARBIER.
}

Observatoire de Marseille.

\begin{abstract}
Sommaire. - Après quelques considérations théoriques sur l'équation intégrale à laquelle conduit la méthode de Fabry et Buisson, on montre comment il est possible de représenter les observations en déterminant la distribution de l'ozone par une série de Charlier du type A. En général on ne peut déterminer que la quantité totale d'ozone et la hauteur moyenne de la distribution, ces quantités átant d'ailleurs l'épaisseur et la hauteur de la couche mince représentative. Exceptionnellement on peut encore calculer la déviation standard de cette distribution. L'étude des données au sol semble indiquer que la distribution est dissymétrique.

Une méthode a été indiquée pour résoudre l'équation relative à la méthode de Cabannes et Dufay. La diffusion par l'atmosphère aux altitudes très grandes joư un rôle prépondérant pour les grandes distances zónithales. Les mesur ss peuvent ètre interprétées à l'aide d'une couche mince donl l'altitude serait voisine de 40 à $50 \mathrm{~km}$, cette altitude n'est d'ailleurs pas, théoriquement tout au moins, l'altitude moyenne de la distribution.

On attire l'attention sur l'intérèt qu'il y aurait à déterminer directement la masse d'air traversée pour chaque dosage d'ozone.

Il semble que le seul moyen possible d'obtenir, d'une manière assez approchée. la distribution de l'ozone soit de combiner la méthode de Fabry et Buisson avec des dosages effectués simultanément à diverses altitudes jusqu'à $15 \mathrm{~km}$ environ.
\end{abstract}

1. Introduction - Il existe deux méthodes pour étudier l'ozone de la haute atmosphère. La première est due à Fabry et Buisson (1). On mesure l'épaisseur d'ozone traversée par un rayon provenant du soleil. Il n'est pas utile d'exposer ici la méthode spectrophotométrique qui permet cette détermination. L'épaisseur d'ozone varie avec la distance zénithale du soleil. En effet soit $R$ le rayon terrestre, $r$ la distance d'un point du rayon lumineux au centre de la terre, $\varphi$ la distance zénithale du soleil et si $\rho(r)$ représente la quantité d'ozone en chaque point de l'atmosphère on trouve qu'un rayon provenant du soleil a traversé une épaisseur totale d'ozone donnée par :

$$
N(\varphi)=\int_{R}^{\infty} \frac{r \rho(r) d r}{\sqrt{r^{2}-R^{2} \sin ^{2} \varphi}}
$$

$N(\varphi)$ est fourni par l'observalion, on se propose de déterminer $\rho(r)$.

Chalonge $\left({ }^{2}\right)$ a étudié un certain nombre de distributions discontinues et il a montré qu'elles salisfont également bien aux observations. Parmi ces distributions l'une d'entre elles a été regardée pendanl longtemps comme devant être très proche de la réalité : elle est connue sous le nom de couche mince, car on suppose tout l'ozone réparti dans une couche mince d'épaisseur $\varepsilon$ située à une distance $D$ du centre de la terre. Cette distribution donnait :

(1) Voir par exemple : Fabry et Buissox. Mémorial des Sciences physiques, XI, 1930. L'absorption des radiations dans la haute atmosphère.

${ }^{2}{ }^{2}$ Journal de Physique 1932, t. 3, p. 21.

$$
N(\varphi)=\frac{\varepsilon D}{\sqrt{D^{2}-R^{2} \sin ^{2} \varphi}} .
$$

Chalonge a montré le premier, comme nous venons de le dire, que cette loi ne s'imposait pas. Une remarque est utile ici. Soit l'équation inlégrale très simple :

$$
\int_{0}^{x} \varphi(s) \mathrm{d} s=f(x)
$$

on sait (1) que la fonction $\varphi(s)$ est indéterminée si on la suppose seulement bornée et intégrable. Si on admet en outre qu'elle est continue et si $f(x)$ est nul pour $x=0$ et admet une dérivée elle est parfaitement bien déterminée. On peut se demander si notre équation (1) n'admet pas une solution continue parfaitement bien déterminée. La première partie de ce travail sera consacrée à l'étude de cette équation et aux conséquences physiques qu'on en peut tirer. Dans la deuxième partie nous examinerons la deuxième méthode de dosage global de l'ozone atmosphérique. Cette méthode a été imaginée par Cabannes et Dufay $(\stackrel{2}{ })$, elle est connue sous le nom de méthode du ciel bleu. Elle consiste à photographier le spectre du ciel au zénith. Suivant l'hypothèse des auteurs, l'ozone devait se trouver audessus des couches diffusantes alors tous les rayons que recevait l'observateur avaient traversé la même quantité d'ozone et on se trouvait ramené à la résolution d'une équation analogue à (1). Malheureusement certaines mesures et en particulier celles de Götz et de Dob-

(1) Gounsat. Analyse, T. 3, p. $33 \%$.

(8) Journal de Physique, 1927, T. 8, p. 125. 
son ne semblent pas s'accorder avec cette hypothèse Chalonge a interprété ces mesures à l'aide du schéma suivant : l'atmosphère est supposée ne plus diffuser audessus de $30 \mathrm{~km}$ d'altitude, l'ozone est réparti en deux couches situées l'une en dessous, l'autre en dessus de cette limite. Nous indiquons en particulier que la limitation de la diffusion aux altitudes inférieures à 30 kms n'est pas légitime.

2. Remarques préliminaires sur l'équation (1). - C'est une équation de Fredholm de première espèce. M. E. Picard $\left({ }^{1}\right)$ a établi une méthode permettant de reconnaître si une telle équation a une solution, et de la former si elle existe. Dans le cas actuel les calculs seraient absolument inextricables. Mineur (2) a montré que si l'équation (1) admel une solution, celle ci est unique.

Rappelons cetle démonstration. Posons :

$$
\frac{1}{r^{2}}=u \quad \frac{1}{R^{2}}=u_{0} \quad \frac{1}{2} r^{3} p(r)=f(u) .
$$

Il vient alors :

$$
\begin{aligned}
N(\varphi)=I_{0}+\frac{1}{2} I_{1} & \sin ^{2} \varphi+\ldots \\
& +\frac{1.3 \ldots(2 n-1)}{2.4 \ldots 2 n} I_{n} \sin ^{2 n} \varphi+\ldots
\end{aligned}
$$

avec :

$$
I_{n} u_{0}{ }^{n}=\int_{0}^{u_{0}} u^{n} f(u) \mathrm{d} u \quad(n=0,1,2 \ldots) .
$$

Le développement de la fonction donnée $N(\varphi)$ suivant les puissances de $\sin ^{2} \varphi$ fera connaître les nombres $I_{0}, I_{1}$, etc.

C'est un théorème classique que si la fonction $f(u)$ est continue on peut la développer en série de polynomes et qu'elle est unique. Pour effectuer ce développement il serait commode par un changement linéaire de variable d'amener les limites à être -1 et $+\mathbf{1}$. Les polynomes seraient alors des polynomes de Legendre pour lesquels on trouve des tables de coefficients $\left({ }^{3}\right)$. Si le développement converge il représente bien $f(u)$ mais rien ne prouve à priori qu'il convergera.

Nous allons montrer maintenant que dans un cas simple, très important en pratique l'équation (1)n'admel pas de solution continue. C'est le cas où $N(\varphi)$ est donné par (2). Nous avons déjà dit que cette expression représente bien les observations. On l'interprète par l'hypo. thèse de la couche mince, mais rien ne prouve qu'une distribution continue ne puisse conduire à la même formule. Posons $\frac{1}{D^{2}}=a$ et développons $N(\varphi)$ suivant les puissances de $\sin ^{2} \varphi$. Les équations (3) deviennent :

(1) Rendiconti del Circolo matematico di Palermo XXIX, 1910. (2) Journal de Physique 1932, Série VII, T. 3, p. 145.

(3) Smithsonian mathématical formule 1922 p. 192.

$$
\begin{aligned}
\varepsilon & =\int_{0}^{u_{0}} f(u) \mathrm{d} u \\
\varepsilon a & =\int_{0}^{u_{0}} u f(u) \mathrm{d} u \\
\varepsilon a^{n} & =\int_{0}^{u_{0}} u^{n} f(u) \mathrm{d} u .
\end{aligned}
$$

Multiplions les deux membres de la première équation par $a$ et retranchons-la de la deuxième. Multiplions-les ensuite par $a^{2}$ et retranchons de la $3^{{ }}$la équation et ainsi de suite. Il vient :

$$
\begin{aligned}
& \int_{0}^{u_{0}}(u-a) f(u) \mathrm{d} u=0 \\
& \int_{0}^{u_{0}}\left(u^{2}-a^{2}\right) f(u) \mathrm{d} u=0 \\
& \int_{0}^{u_{0}}\left(u^{n}-a^{n}\right) f(u) \mathrm{d} u=0 .
\end{aligned}
$$

Posons $(u-a) f(u)=f_{1}(u)$. Il vient, car $a$ est essentiellement positif

$$
\int_{0}^{u_{0}} u^{n} f_{1}(u) \mathrm{d} u=0 \quad(n=0,1,2 \ldots) .
$$

On sait qu'un tel système ne peut être vérifié que par

$$
f_{1}(u) \equiv 0
$$

mais $f(u)$ ne peut être identiquement nul et par suite l'équation proposée n'admet pas de solution continuc. Le développement de polynomes qu'on aurait obtenu aurait divergé. Si la fonction $N(\varphi)$ était un peu différente de (2) il pourait peut-être y avoir une solution continue mais le développement serait très peu convergent et ne se prêterait pas aux calculs numériques.

On montre sans peine que pour l'expression suivante

$$
\begin{array}{r}
N(\varphi)=\frac{\varepsilon_{1} D_{1}}{\sqrt{D_{1}^{2}-R^{2} \sin ^{2} \varphi}}+\frac{\varepsilon_{2} D_{2}}{\sqrt{D_{2}^{2}-R^{2} \sin ^{2} \varphi}}+\ldots \\
\quad+\frac{\varepsilon_{n} D_{n}}{\sqrt{D_{n}^{2}-R^{2} \sin ^{2} \varphi}}
\end{array}
$$

l'équation (1) n'a pas non plus de solution continue.

Faisons encore une remarque très importante pour la suite : soit une équation de Fredholm de première espèce

$$
f(x)=\int_{a}^{b} K(x, s) h(s) \mathrm{d} s
$$

qui n'admette pas de solution. On montre $\left(^{1}\right)$ que sous des conditions assez générales on peut trouver une fonction $h(x)$ que telie $\int_{a}^{b} K(x, s) h(s) \mathrm{d} s$ diffère en moyenne aussi yeu qu'on le voudra de $f(x)$. La fonction $f(x)$ étant de nature expérimentale n'est définie qu'avec une certaine approximation et une solution exacte en moyenne aura la même signification qu'une solution au sens strict du mot. Ilest facile deformer pour l'équation (1) une telle solution lorsque $N(\varphi)$ a l'expression (2) Soit :

(1) Godrgat. Analyse, T. 3, p. 179. 


$$
p(r)=\frac{\varepsilon}{\sigma \sqrt{2 \pi}} \mathbf{e}^{-\frac{(r-D)^{2}}{2 \sigma^{2}}}
$$

l'intégrale

$$
\frac{\varepsilon}{\sigma \sqrt{2 \pi}} \int_{R}^{\infty} \frac{r \mathbf{e}^{-\frac{(r-D)^{2}}{2 \sigma^{2}}} \mathrm{~d} r}{\sqrt{r^{2}-R^{2} \sin ^{2} \varphi}}
$$

tend vers

$$
\frac{\varepsilon D}{\sqrt{D^{2}-R^{2} \sin ^{2} \varphi}}
$$

lorsyue $\sigma$ tend vers zéro. Par suite il sufiira de prendre $\sigma$ assez petit pour que (1) diffère aussi peu qu'on le voudra de (2).

Nous allons maintenant nous occuper de la formalion pratique de ces solutions.

3. Méthode pratique pour la résolution de l'équation (1). - La méthode que nous proposons permet d'exprimer $\rho(r)$ à l'aide d'un développement qui en général sera très convergent.

Nous allons utiliser le fait que dans la pratique $N(\varphi)$ ne différera jamais beaucoup de son expression (2). Effectuons le changement de variable.

$$
\sin \psi=\frac{R}{D} \sin \psi
$$

alors $N(\psi)$ sera peu différent de $\varepsilon \sec \psi$.

Posons encore

$$
r-D=x
$$

l'équation (1) devient

$$
N^{\top}(\psi)=\int_{R-D}^{\infty} \frac{p(x)[D+x] \mathrm{d} x}{\sqrt{D^{2} \cos ^{2} \psi+2 D x+x^{2}}}
$$

Pour que $\frac{1}{\sqrt{D^{2} \cos ^{2} \psi+2 D x+x^{2}}}$ puisse être développé suivant les puissances de $x$, il faut que :

$$
\left|\frac{2 D x+x^{2}}{D^{2} \cos ^{2} y}\right|<1
$$

c'est-à-dire :

$x<x_{0} \quad$ avec $\quad x_{0}=D\left[\sqrt{2-\sin ^{2} \cdot}-1\right] \sim D-R \sin \varphi$.

Posons

$$
h=D-R
$$

$h$ est l'altitude de la couche mince représentative qu'on sait être de l'ordre de $50 \mathrm{~km}$. On voit que si les mesures vont jusqu'à $90^{\circ}$ on a $x_{\mathrm{v}}=50 \mathrm{~km}$ (c'est-à-dire une altitude de $100 \mathrm{~km}$ ). Ecrivons

$$
\begin{aligned}
I^{\prime}(\psi)= & \int_{R-D}^{x_{0}} \frac{(D+x) \rho(x) \mathrm{d} x}{\sqrt{D^{2} \cos ^{2} \psi+2 D x+x^{2}}} \\
& \quad+\int_{x_{0}}^{\infty} \frac{(D+x) \rho(x) \mathrm{d} x}{\sqrt{D^{2} \cos ^{2} \downarrow+2 \nu x+x^{2}}} .
\end{aligned}
$$

La cleuxième intégrale sera toujours très petite $\operatorname{car} \rho(x)$ doit tendre rapidement vers zéro aux grandes altitudes. Soit $J$ sa valeur on aura toujours :

$$
J<\frac{D+x_{0}}{\sqrt{D^{2} \cos ^{2} \psi+2 D x_{0}+x_{0}^{2}}} \int_{x_{0}}^{\infty} \rho(x) \mathrm{d} x .
$$

En développant la première intégrale suivant les puissances de $x$ il vient :

$$
\begin{aligned}
N(\psi) & =\sec \psi \int_{R-D}^{x_{0}} \rho(x) \mathrm{d} x-\frac{\sec \psi \operatorname{tg}^{2} \psi}{D} \int_{R-D}^{x_{0}} x \rho(x) \mathrm{d} x \\
& +\frac{3}{2} \frac{\sec ^{3} \psi \operatorname{tg}^{2} \psi}{D^{2}} \int_{R-D}^{x_{0}} x^{2} \rho(x) \mathrm{d} x+\ldots+J
\end{aligned}
$$

Par un calcul d'identification nous mettrons la fonction expérimentale $N(\psi)$ sous la forme :

$N(\psi)=m_{0} \sec \psi-\frac{m_{1}}{D} \sec \psi \lg ^{2} \psi+\frac{3}{2} \frac{m_{2}}{D^{2}} \sec ^{3} \psi \operatorname{tg}^{2} \psi+\ldots$

Supposons d'abord $J$ négligeable ce qui sera presque toujours le cas en pratique, on obtient :

$$
\left.\begin{array}{l}
m_{0}=\int_{R-D}^{x_{0}} \rho(x) \mathrm{d} x \\
m_{1}=\int_{R-D}^{x_{0}} x \rho(x) \mathrm{d} x \\
m_{2}=\int_{R-D}^{x_{0}} x^{2} \rho(x) \mathrm{d} x
\end{array}\right\}
$$

Nous allons représenter $\rho(x)$ par une série de Charlier de type A.

$$
F(x)=\frac{m_{0}}{\sigma}\left[F_{0}(x)+\beta_{3} F_{3}(x)+\beta_{4} F_{4}(x)+\ldots\right]
$$

avec

$$
\begin{gathered}
F(x)=\frac{1}{\sigma \sqrt{2 \pi}} \mathrm{e}^{-\frac{\left(x-b^{2}\right.}{2 \sigma^{2}}} \\
F_{0}=\sigma F(x) \quad F_{3}=\sigma^{4} F^{\mathrm{II}} \quad F_{4}=\sigma^{5} \boldsymbol{F}^{\mathrm{IV}} .
\end{gathered}
$$

Ecrivons encore les équations (7) sous la forme

$$
\begin{aligned}
m_{0}+\int_{-\infty}^{R-D} \rho(x) \mathrm{d} x & +\int_{x_{0}}^{+\infty} \rho(x) \mathrm{d} x \\
& =\int_{-\infty}^{+\infty} \rho(x) \mathrm{d} x \\
m_{1}+\int_{-\infty}^{R-D} x \rho(x) \mathrm{d} x & +\int_{x_{0}}^{\infty} x \rho(x) \mathrm{d} x \\
& =\int_{-\infty}^{+\infty} x \rho(x) \mathrm{d} x \\
m_{2}+\int_{-\infty}^{R-D} x^{2} \rho(x) \mathrm{d} x & +\int_{x_{0}}^{\infty} x^{2} \rho(x) \mathrm{d} x \\
& =\int_{-\infty}^{+\infty} x \rho(x) \mathrm{d} x
\end{aligned}
$$

Les intégrales qui figurent dans les premiers membres sont toujours très petites et pratiquement négligeables. 
Remplaçons-les par zéro; on obtient ainsi une première approximation pour $p(x)$ par les formules bien connues ( 1 ):

$$
\begin{gathered}
m_{s}^{\prime}=\frac{m_{s}}{m_{0}} ; \quad b=m_{1}^{\prime} ; \quad \sigma^{2}=m_{2}^{\prime}-b^{2} \\
\beta_{3}=-\frac{\nu_{3}}{6 \sigma^{3}} ; \quad \beta_{4}=\frac{1}{24}\left[\frac{\nu_{4}}{\sigma^{4}}-3\right]
\end{gathered}
$$

avec

$$
\begin{aligned}
& v_{3}=m^{\prime}{ }_{3}-3 b m_{2}^{\prime}+2 b^{3} \\
& v_{4}=m_{4}^{\prime}-4 b m^{\prime}{ }_{3}+6 b^{2} m_{2}^{\prime}-3 b^{3} .
\end{aligned}
$$

Si l'on juge nécessaire de tenir compte des intégrales des premiers membres des équations (9) on y remplacera $\rho(x)$ par sa première approximation. Le catcul sera facile et on redéterminera $\rho(x)$ avec les premiers membres corrigés. Une fois qu'on a obtenu $\rho(x)$ on détermine une limite supérieure de $J$ grâce à (̋). Si on juge que cette valeur est supérieure aux erreurs d'observations il faudra calculer $J$.

$$
J=\int_{r_{0}}^{\infty} \frac{r \rho(r) d r}{\sqrt{r^{2}-R^{2} \sin ^{2} \varphi}} .
$$

Nous faisons le changement de variable :

$$
\sin \psi_{1}=\frac{R}{D_{1}} \sin \varphi
$$

et on prendra pour $D_{1}$, une valeur supérieure à $r_{0}$. On obtiendra un développement analogue à $(6)$. On calculera alors $J$ en fonction de $\psi$ et on corrigera en conséquence $N(\psi)$. On reprendra alors comme ci-dessus le calcul de $p(x)$.

Tout ceci peut sembler un peu long mais comme nous l'avons dit, et nous insistons sur ce point, $J$ et les intégrales des premiers membres des équations (9) seront pratiquement toujours négligeables.

4. Etude d'un cas particulier important. Nous supposons que les observations puissent être représentées par un seul terme.

$$
N(\psi)=\varepsilon \sec \psi \cdot
$$

Presque toutes les séries d'observations qu'on possède peurent être représentées par une telle formule. L'angle $\psi$ est défini par (4) mais la constante $D$ qui figure dans cette formule n'est pas forcément la même pour toutes les séries. En vertu des équations (7) on a alors

(1) Pour ces formules at tout ce qui concerne les fonctions du type A nous renvoyons au mémoire fondamental de Charlier où on trouvera des tables numériques. Lunds Universitets Arsskrıft N. B Afd 2, 1906 Bd $1 \mathrm{Nr}$ 3. Ou Meddelanden fran Lunds Observat) rium. Série II n ${ }^{\circ}$ 4. On peut encore consulter: Statistique Mathématique par G. Darmors. (Doin, Edit. Paris 1928). Rappelons qu'une répartition se laisse pratiquement toujours représenter par une série telle que (8) à trois termes Une telle distribution peut d'ail leurs se représenter par la superposition de deux courbes normales de Gauss. $b$ s'appelle la moyenne, $\sigma$ la déviation standard, $\beta_{3}$. le coeflieient de dissymétrie et $\beta_{*}$ l'excès.

$$
\int \rho(x) \mathrm{d} x=\varepsilon
$$

$\varepsilon$ représente bien la quantité totale d'ozone, résultat évident à priori. On a ensuite :

$$
\int x p(x) \mathrm{d} x=0
$$

c'est-à-dire $b=o$. La valeur moyenne de $x$ est zéro ce qui veut dire que la valeur moyenne de $r$ est $D$ ou encore que l'altitude moyenne (ou centre de gravité) de la distribution est l'altitude $h=D-R$ de la couche mince représentative. Pour préciser davantage la différence entre la valeur moyenne de $r$ et $D$ est indiscernable par l'observation.

La troisième des équations (7) donne

$$
\int x^{2} p(x) \mathrm{d} x=0
$$

c'est à-dire $\sigma=0$ ou tout au moins $\sigma$ assez voisin de zéro pour qu'on ne puisse pas mettre en évidence par l'observation le terme $\sec ^{3} \psi \operatorname{tg}^{2} \psi$. Précisons ce point en déterminant la plus grande valeur que puisse atteindre $\sigma$ pour satisfaire à cette condition. Le deuxième terme du développement de $N(\psi)$ étant nul on a :

$$
N(\psi)=\varepsilon \sec \psi+\frac{3}{2} \frac{m_{2}}{D^{2}} \sec ^{3} \psi \operatorname{tg}^{2} \psi .
$$

Nous admettons que pour qu'on puisse déterminer le terme en $\sec ^{3} \psi \operatorname{tg}^{2} \psi$ sa valeur doit atteindre les $2 / 100$ de la valeur du premier terme pour $\rho=90^{\circ}$ (c'est-à-dire pour $\psi=80^{\circ}$ si l'on suppose $\mathrm{h}=50 \mathrm{~km}$ )

$$
\frac{3}{2} \frac{m_{3}}{D^{2} \varepsilon} \sec ^{2} \psi \operatorname{tg}^{2} \psi>\frac{2}{100}
$$

d'où

$$
m_{2}^{\prime}=\frac{m_{2}}{\varepsilon}>450
$$

par suite $\sigma^{2}>450$ ou encore $\sigma>21$.

Pour toute distribution telle que $\sigma<21$ le terme en $\sec ^{3} \psi \operatorname{tg}^{2} \psi$ ne pourra être détecté par l'observation. Cette valeur limite de $\sigma$ varie d'ailleurs avec l'altitude moyenne de la distribution.

Pour préciser la signification de cette limite calculons pour $h=50 \mathrm{kms}$ et $\sigma=21$ entre quelles altitudes se trouvent la moitié et les 9/10 de l'ozone. En limitant $p(x)$ à son premier terme on trouve que la moitié de l'ozone se trouve entre 36 et $64 \mathrm{kms}$ et les $9 / 10$ entre $1 \mathrm{a}$ et $8 \% \mathrm{kms}$. On voit que la dispersion permise par la valeur limite de $\sigma$ est assez considérable. On pourrait d'une manière tout à fait analogue déterminer des limites pour les coefficients $\beta_{3}$ et $\beta_{4}$. Elles sont très écartées. Pour nous résumer :

Lorsque les mesures se laissent interpréter par une couche mince d'épaisseur $\varepsilon$ et d'altitude h cette épaisseur est la quantité totale d'ozone et h est la hauleur moyenne de la distribution. La déviation standard o peut prendre n'importe quelle valeur comprise entre zéro et une certaille limite fanction de h. Le deuxième et le troisième 
terme de $\rho(x)$ sont complètement indéterminés ainsi d'ailleurs que les suivants.

๖. Discussion des mesures de Gáuzit. - Dernièrement (1) Gauzit a obtenu des séries d'observations qui ne se laissaient pas représenter par une loi simple en sec $\downarrow$. Il a interprété ses résultats par la superposition d'une couche mince et d'une distribution dans toute l'atmosphère à concentration constante. Voici les chiffres relatifs à trois journées diffé. rentes $\left({ }^{2}\right)$.

\begin{tabular}{|c|c|c|}
\hline DATE & $\begin{array}{c}\text { ALTITUde } \\
\text { DE LA COUCHE } h\end{array}$ & $\begin{array}{l}\text { PROPORTIOX D'OZONE } \\
\text { DIFFUS } p\end{array}$ \\
\hline 8 août 1933 . & 21 & 23 pour 100 \\
\hline ût. . . . . . & 23 & 30 pour 100 \\
\hline 28 août. . . . . . . . & 48 & 8 pour 100 \\
\hline
\end{tabular}

Ce schéma a le défaut de ne pas rendre très bien compte des dosages effectués au voisinage du sol. D'après des recherches récentes $\left(^{3}\right)$ il semble que la quantité d'ozone croisse avec l'altitude alors que le schéma de Gauzit conduit à un résultat inverse. Nous préférons interpréter ces nombres par une distribution continue que nous limiterons à son premier terme. On calcule les quantités $\int x p(x) d x$ et $\int x^{2} p(x) d x$ et on obtient les résultats suivants :

\begin{tabular}{crr}
$\stackrel{h}{\text { Date }}$ & \multicolumn{1}{c}{} & \multicolumn{6}{c}{} \\
18 août $\ldots \ldots$ & 17,6 & 9,5 \\
23 août $\ldots \ldots$ & 18,2 & 11,7 \\
28 août $\ldots \ldots$ & 44,8 & 15,6
\end{tabular}

Il est à remarquer que l'altitude moyenne des distributions obtenues par Gauzit est beaucoup plus faible que la valeur voisine de $50 \mathrm{kms}$ qu'on obtient habituellement : Le rapport $\frac{\sigma}{h}$ a les valeurs $0, .44 ; 0,63$ et 0,36 . On peut se demander si à la précision des mesures près il ne reste pas constant et voisin de $0, \stackrel{30}{0}$.

6. Conditions au sol. - Nous venons de voir qu'on ne peut que toul à fait exceptionnellement déterminer le premier terme du développement de $\rho(x)$ alors qu'il en faudrait trois pour avoir une bonne représentation de la distribution de l'ozone en altitude. On reconnaît sans peine que les mesures devraient êlre environ 1000 fois plus précises pour permettre leur calcul et ceci est tout à fait impossible à réaliser. Pour obtenir de nouveaux renseignements sur la distribu. tion de l'ozone on peut songer à faire appel à des dosages effectués au sol à différentes altitudes. Ce

(1) C. R. 193', T. 198, p. 492.

(2) Nous avons ramené les altitudes à être comptées à partir du niveau de la mer.

(3) Gotz et Maier Leibnitz, Zeilschfrit fuir Geophysıli 1933,9, p. 253 et Chalonge, Vassy, Barbier. Bulletin de la Soctété françatse de Physique $1933, \mathbf{n}^{\circ} 347$, p. 155 . procédé est évidemment assez aléatoire car la distribution de l'ozone peut être troublée dans la troposphère ou au voisinage du sol.

Supposons $\rho(x)$ limité à son premier terme.

$$
f(x)=\frac{\varepsilon}{\sigma \sqrt{2 \pi}} e^{-\frac{x^{2}}{2 \sigma^{2}}}
$$

Si $z$ est l'altitude d'un point de l'atmosphère.

$$
z=x+h \text {. }
$$

$h$ étant l'altitude moyenne de la distribution, on a au sol

$$
\begin{aligned}
\rho_{0} & =\frac{\varepsilon}{\sigma \sqrt{2 \pi}} \mathbf{e}^{-\frac{h^{2}}{2 \sigma^{2}}} \\
\left(\frac{1}{\rho} \frac{\mathrm{d} \rho}{\mathrm{d} z}\right)_{0} & =\frac{h}{\sigma^{2}}
\end{aligned}
$$

\begin{tabular}{|c|c|c|c|c|c|}
\hline$\sigma \ldots \ldots \ldots$ & 10 & 20 & 30 & 40 & 50 \\
\hline $10^{4} p_{0} \ldots \ldots$ & 0 & 3 & 10 & 14 & 10 \\
\hline$\left(\frac{1}{\rho} \frac{d \rho}{d z}\right)_{0} \ldots$ & $0 . \check{5}$ & 0,12 oั & 0,050 & 0,031 & 0,020 \\
\hline
\end{tabular}

Les mesures donnent en moyenne $\left(^{1}\right) p_{0}=20.10^{-4} \mathrm{~cm}$. Prenons $s=0,3 \mathrm{~cm} h=50$ et pour diverses valeurs de $\sigma$ calculons $\rho_{0}$ et $\left(\frac{1}{\rho} \frac{\mathrm{d} \rho}{\mathrm{d} z}\right)_{0}$. On trouve

On constate que :

a) Aucune valeur de $\sigma$ ne permet de retrouver la valeur expérimentale de $\rho_{0}$. Les grandes valeurs de $\sigma$ conduisent à des valeurs qui en diffèrent assez peu, mais elles ne sont pas acceptables car elles seraient mises en évidence par les observations (il faut que $\sigma$ soit plus petit que 21).

b) D'après les mesures de Götz et Maier Leibniz $f_{0}=20 \cdot 10^{-4}$ à $500 \mathrm{~m}$ d'altitude et $30,10^{-4}$ à 2000 , c'est-à-dire

$$
\left(\frac{1}{p} \frac{d p}{d z}\right)_{0}=0,3 .
$$

L'accord serait convenable pour les petites valeurs de $\sigma$. Si on admet qu'il n'y a pas d'anomalies dans la répartition de l'ozone au voisinage du sol, ces résultats montreraient que la distribution ne peut être représentée par une courbe de Gauss el qu'il est nécessaire de faire intervenir le deuxième terme du développement, celui qui permet de représenter la dissymétrie de la courbe.

Calculons encore les valeurs de $p_{0}$ et de $\left(\frac{1}{\rho} \frac{d p}{d z}\right)_{0}$ auxquelles conduiraient les mesures de Gauzit dans le cas où $\rho(x)$ est limité à son premier terme. Il vient :

(1) Götz et Maier Leibnitz. Loe cit., et Buisson, Jausserar RovakD. Revue d'optique 1933, 12, p 70. 


$$
\begin{array}{cccc}
\text { Date........ } & 18 \text { août } & 23 \text { aoùt } & 28 \text { aoùt } \\
10^{\prime} p_{0} \ldots \ldots \ldots & 12 & 36 & 1 \\
\left(\frac{1}{\rho} \frac{\mathrm{d} \rho}{\mathrm{d} z}\right)_{0} \ldots \ldots & 0,19\lrcorner & 0,133 & 0,18 \check{ }
\end{array}
$$

Pour le 18 et le 23 août les résultats sont acceptables tout au moins en ce qui concerne $\rho_{0}$. Ainsi la dissymétrie de la répartition serait moins accentuée lorsque son attitude moyenne s'abaisse.

7. Remarques diverses sur la méthode de Fabry et Buisson. - Dans tout ce qui précède on a supposé que l'absorption par l'ozone est indépendante des conditions physiques dans lesquelles se trouve ce gaz. Tout récemment Chalonge (1) a indiqué que Madame Lefebvre reprenant les recherches de Wulf et Melvin a trouvé que pour certaines longueurs d'onde les coefficients d'absorption seraient très différents aux basses températures de ce qu'ils sont aux tempétures habituelles. Nous allons très brièvement donner quelques indications sur les conséquences de cet effet en ce qui concerne la détermination de la répartition de l'ozone. L'équation (1) devient

$N(\varphi)=\frac{1}{K_{0}-K_{0}^{\prime}} \int_{R}^{\infty}\left(K-K^{\prime}\right) \frac{r \rho(r) \mathrm{d} r}{\sqrt{r^{2}-R^{2} \sin ^{2} \varphi}}$

$K$ et $K^{\prime}$ sont les cofficients d'absorption de l'ozone pour deux longueurs d'ondes. Leur différence est fonction de la température qui est elle-mème fonction de l'altitude. $K_{0}$ et $K_{0}^{\prime}$ représentent ces coefficients mesurés suus les conditions normales. Passons à la variable $x$.

$$
x=r-D
$$

et posons

$$
\frac{K-K^{\prime}}{K_{0}-K_{0}^{\prime}}=k(x)
$$

Ce qu'on détermine n'est plus $\rho(x)$ mais $\rho(x) \times k(x)$. Comme $k(x)$ est connu $\left({ }^{2}\right)$ on en déduit $\rho(x)$.

Supposons que la résolution de (12) nous ait conduit à la solution :

$$
\rho(x) k(x)=\frac{\varepsilon}{\sigma \sqrt{2 \pi}} \Theta^{-\frac{x^{2}}{2 \sigma^{2}}} .
$$

Supposons encore à priori que $\rho(x)$ puisse être mis sous la forme :

alors on a :

$$
P(x)=\frac{\varepsilon_{1}}{\sigma_{1} \sqrt{2 \pi}} \mathbf{e}^{-\frac{\left(x-b_{1}\right)^{2}}{2 \sigma_{1}{ }^{2}}}
$$

$$
\begin{aligned}
k(x) & =\frac{\varepsilon \sigma_{1}}{\xi_{1} \sigma} \mathrm{e}^{-\frac{1}{2}\left[\frac{x^{2}}{\sigma^{2}}-\frac{(x-b)^{2}}{\sigma_{1}^{2}}\right]} \\
& =\frac{\varepsilon \sigma_{1}}{\varepsilon_{1} \sigma} \mathrm{e}^{-\frac{1}{2}\left(\frac{1}{\sigma^{2}}-\frac{1}{\sigma_{1}^{2}}\right)\left[\left(x+\frac{b \sigma^{2}}{\sigma_{1}^{2}-\sigma^{2}}\right)^{2}-\frac{l^{2} \sigma_{1}^{2} \sigma^{2}}{\left(\sigma_{1}^{2}-\sigma^{2}\right)^{2}}\right] .} .
\end{aligned}
$$

(1) Bulletin de la Sociélé française de Physique 1934, $\mathrm{n}^{\circ}$ 349, p. $16 . \mathrm{S}$.

(2) En admettant qu'on connaisse la variation de la température avec l'altitude.
Si on veut que la hauteur moyenne de la distribution vraie qui est définie par (14) soit la mème que celle qu'on aurait déduite de (13) en supposant $k=1$, c'està-dire l'absorption par l'ozone indépendante de la température, il faut que $b$ soit nul. On voit alors que $k(x)$ prend la forme

$$
k(x)=\frac{\varepsilon \sigma_{1}}{\varepsilon_{1} \sigma} \mathrm{e}^{-\frac{1}{2}\left(\frac{1}{\sigma^{2}}-\frac{1}{\sigma_{1}^{2}}\right) x^{2}}
$$

Par suite $k(x)$, suivant le signe de $\frac{1}{\sigma^{2}}-\frac{1}{\sigma_{1}{ }^{2}}$, passe par un maximum ou par un minimum pour $x=0$ c'est-à-dire pour la hauteur moyenne de la distribution d'ozone. Ceci ne doit pas être très éloigné de la réalité car on a tout lieu de croire que la température de l'atmosphère passe par un maximum pour cette valeur de l'altilude. Tout ceci est très schématique mais on peut dire qu'étant donné le peu de précision que comporte la résolution de l'équation (1) il est tiès probable qu'on n'aura pas à craindre, dans le cas de variation de l'absorption par l'ozone avec la température, de grosses erreurs sur la hauteur moyenne de la distribution mais seulement sur la quantité totale d'ozone et sur sa déviation standard.

Remarquons encore, qu'au moins en théorie, il est possible de déterminer expérimentalement la répartition des températures dans l'atmosphère. A l'équation 12) joignons une équation identique mais valable pour deux autres longueurs d'ondes et posons

$$
\frac{K^{\prime \prime}-K^{\prime \prime \prime}}{K_{0}^{\prime \prime}-K_{0}^{\prime \prime \prime}}=k_{1}(x) \text {. }
$$

Les solutions de ces deux équations sont $k(x) \times \rho(x)$ et $k_{1}(x) \times \rho(x)$. Leur rapport est indépendant de la répartition de l'ozone dans l'atmosphère et est fonction uniquement de l'altitude. Par des mesures au laboratoire on peut exprimer ce rapport en fonction de la température. L'identification de ces deux expressions du rapport fait alors connaître la température en fonction de l'altitude.

Un autre point sur lequel il convient d'attirer l'attention est le suivant : Pour déterminer $N(\varphi)$ d'après les observations on a l'équation :

$$
\log \frac{I}{I^{\prime}}=\mathrm{C}^{\mathrm{te}}-\left(d-d^{\prime}\right) m_{0}-\left(\boldsymbol{K}-\boldsymbol{K}^{\prime}\right) N(\varphi) .
$$

$\frac{I}{I^{\prime}}$ est le rapport des intensités mesurées pour deux longueurs d'ondes, $m_{0}$ est la masse d'air traversé par un rayon arrivant sous la distance zénithale $\varphi$. $d$ et $d^{\prime}$ sont les coefficients d'absorption de l'air pour les deux longueurs d'ondes utilisées.

La constante est déterminée par extrapolation.

Habituellement on prend la valeur de $m_{0}$ dans une table. Nous pensons qu'il serait bien préférable de déterminer expérimentalement sa valeur en même temps qu'on effectue les observations relatives à l'ozone. Il faudrait posséder un deuxième spectro- 
graphe permettant de travailler dans une région du spectre visible où il n'y aurait pas d'absorption sélective par l'ozone $\left(\boldsymbol{K}-\boldsymbol{K}^{\prime}=\mathbf{0}\right)$ ni d'ailleurs par un autre gaz. En effet les tables de $m_{0}$ sont purement théoriques et les astronomes ont constaté depuis longtemps déjà que même en l'absence de toute brume et de toutes poussières leur emploi esı hasardeux, mème aux faibles distances zénithales. Il est probable qu'au voisinage de l'horizon on s'expose à des erreurs très considérables. Ceci peut provenir en particulier comme l'a montré M. Rougier ( ${ }^{1}$ ) de nuages invisibles. Aux grandes distances zénithales une telle couche de nuages invisibles serait traversée par un rayon suivant un chemin très long. En outre le rayon est encore dans la troposphère en des points très éloignés de l'observa. teur où les conditions météorologiques peuvent ètre très différentes de celles qu'il observe. Les observateurs, et Gauzit en particulier $\left({ }^{2}\right)$, ont parfois rencontré des séries tout à fait anormales et ils ont attribué ces anomalies à l'ozone, à notre avis il est plus probable qu'elles soient d'ordre troposphérique. La méthode que nous préconisons aurait en outre l'avantage de permettre d'éliminer des séries franchement mauvaises qui se reconnaîtraient à la marche irrégulière de $m_{\hat{0}}$ en fonction de $\varphi$.

Signalons encore une conséquence de ce qui a été dit précédemment. Supposons qu'il y ait deux couches d'ozone, ou plus généralement que la distribution d'ozone soit la superposition de deux répartitions ayant des natures dîfférentes dont l'une par exemple serait stratosphérique et l'autre troposphérique. Soient $\varepsilon_{1}$ et $\varepsilon_{2}$ les quantités à'ozone appartenant à chaque distribution et soient $h_{1}$ et $h_{2}$ leurs altitudes. L'observation fera connaître la quantité tolale $\_$et la hauteur moyenne $h$ de la distribution résultante. On aura

$$
\begin{aligned}
\varepsilon & =\varepsilon_{1}+\varepsilon_{2} \\
\varepsilon h & =\varepsilon_{1} h_{1}+\varepsilon_{2} h_{2}
\end{aligned}
$$

Si nous supposons que trois des quantités $\varepsilon_{1}, \varepsilon_{2}, h_{1}, h_{2}$ ne varient pas d'un jour à l'autre on aura une relation entre $\varepsilon$ et $h$. Si nous supposons que ces trois quantités varient très peu par rapport à la quatrième on aura seulement corrélation entre $\varepsilon$ et $h$.

8 La méthode de Cabannes et Dufay. - Nous en arrivons à la deuxième méthode d'étude de l'ozone atmosphérique. Comme nous l'avons déjà dit cette méthode consiste à étudier au zénith la lumière diffusée par l'atmosphère. On détermine le rapport des intensités reçues pour deux longueurs d'onde et de la variation de ce rapport avec la dislance zénithale du soleil on cherche à déduire la répartition de l'ozone.

Nous allons mettre le problème en équation en négligeant les diffusions d'ordre supérieur au premier. Il est difficile de reconnaître jusqu'à quel point cette simplification est légitime, aussi les résultats ne

(1) Thèse. Strasbourg 1933, p. 89.

(c) Loc. cit. devront ètre considérés que comme une première approximation.

Considérons un rayon monochromatique de longueur d'onde ^ et d'intensité $I_{0}$ à son entrée dans l'atmosphère terrestre. Nous allons calculer quelle en est la fraclion diffusée vers l'observateur suivant la verticale. Nous effectuerons ici ce calcul très sommairement car il a déjà été effectué par Chalonge $\left(^{1}\right)$ pour des distributions discontinues et les modifications à y apporter pour une distribution continue sont peu importantes.

Soit donc (fig. 1) 0 l'observateur, $0 z$ la verticale, SM un rayon rencontrant la verticale à la cote $z$. Le rayon est affaibli par trois causes.

1. Par l'ozone rencontré;

$2^{\circ}$ Par l'absorption due à la diffusion moléculaire;

$3^{\circ}$ En $M$ une partie seulement est diffusée vers l'observateur.

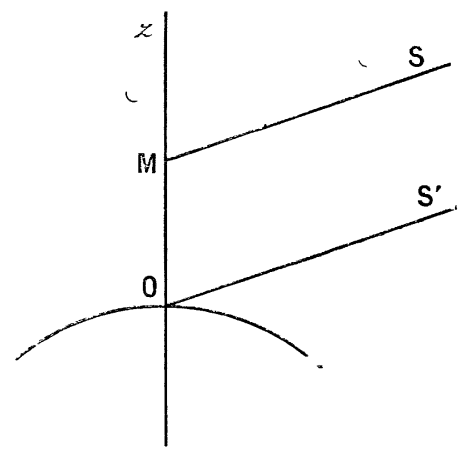

Fig. 1.

L'absorption par l'ozone. Soit $K$ le coefficient d'absorption et $N(\xi)$ la quantité d'ozone rencontrée. L'intensité est multipliée par

$$
e^{-K \Lambda}
$$

et on a d'ailleurs :

$$
N(\varphi)=\int_{\Omega}^{R+z} f(r) \mathrm{d} r+\int_{R+z}^{\infty} \frac{r \xi(r) \mathrm{d} r^{2}}{\sqrt{r^{2}-(R+z)^{2} \sin ^{2} \varphi}}
$$

L'absorption par diffusion sera en appelant $d$ le coefficient d'absorption et $m$ la masse d'air traversée :

$$
\mathbf{e}^{-m d}
$$

$m$ est la somme des masses suivant OM et MS. On prend pour unité la masse suivant $o z$ et on appelle $m_{0}$ la masse suivant $0 S^{\prime}$ parallèle à MS on a :

$$
m=1-\mathbf{e}^{-A z}+m_{0} \mathbf{e}^{-A z}
$$

où $A$ est une constante.

Enfin la proportion de lumière diffusée vers l'observateur est :

$$
B p_{0} \mathbf{e}^{-\boldsymbol{A} z}\left(1+\cos ^{2} \varphi\right)
$$

(1) Loc cit, p. 2. 
$B$ et $p_{0}$ sont des constantes. Il vient donc pour ce rayon

$i=I_{0} B p_{0}\left(1+\cos ^{2} \varphi\right) \mathbf{e}^{-A z} \mathbf{e}^{\left.-d\left[1+{ }^{\prime} m_{0}-1\right) \mathbf{e}^{-A z}\right]} \mathbf{e}^{-K \lambda}$

et la quantité totale de lumière qu'on reçoit est :

$I=\int_{0}^{\infty} i d$

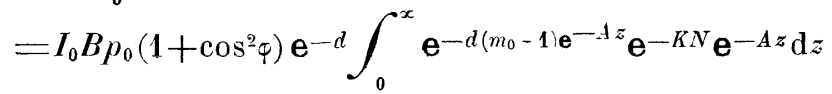

de même pour une autre longueur d'onde $\lambda^{\prime}$

$$
\begin{aligned}
I^{\prime}=I_{0}^{\prime} B^{\prime} p_{0}\left(1+\cos ^{2} \varphi\right) \mathbf{e}^{-d^{\prime}} & \int_{0}^{\infty} \mathbf{e}^{-d^{\prime}\left(m_{0}-1\right) \mathbf{e}^{-A z}} \mathbf{e}^{-K^{\prime} N} \mathbf{e}^{-A z} \mathrm{~d} z .
\end{aligned}
$$

Nous posons: $\quad \mathbf{e}^{-A z}=y$

et il vient

$$
\lg \frac{I}{I^{\prime}}=\lg \frac{\int_{0}^{\prime} \mathbf{e}^{-K N} \mathbf{e}^{-d\left(m_{0}-1\right) y} \mathrm{~d} y}{\int_{0}^{\prime} \epsilon^{-K^{\prime} N} \mathbf{e}^{-d^{\prime}\left(m_{0}-1\right) y} \mathrm{~d} y}+\mathrm{C}^{\mathrm{te}} .
$$
(16).

Il faut déterminer $\rho(r)$ à l'aide des équations $(15$ ! et

\section{Indication dune méthode permettant de} déterminer $p(r)$. - Ecrivons l'équation (1亏) sous la forme équivalente :

$$
N^{r}=N_{0}+N_{1}+N_{2}
$$

avec

$$
\begin{aligned}
& N_{0}^{r}=\int_{R}^{\infty} \frac{r \rho(r) \mathrm{d} r}{\sqrt{r^{2}-R^{2} \sin ^{2} \varphi}} \\
& N_{1}=\int_{R}^{R+z} \rho(r)\left[1-\frac{r}{\sqrt{r^{2}-R^{2} \sin ^{2} \varphi}}\right] \mathrm{d} r \\
& N_{2}=\int_{R+z}^{\infty} r \rho(r)\left[\frac{1}{\sqrt{r^{2}-(R+z)^{2} \sin ^{2} \varphi}}\right. \\
& \left.-\frac{1}{\sqrt{r^{2}-R^{2} \sin ^{2} \varphi}}\right] \mathrm{d} r .
\end{aligned}
$$

Supposons d'akord comme Cabannes et Dufay que l'ozone soit tout entier au-dessus des couches diffusantes limitées à une certaine altitude $z^{\prime}$ (correspondant â une valeur $y^{\prime}$ de $y$ ). Ceci veut dire que pour les valeurs de $z$ inférieures à $z^{\prime}, \rho(r)$ est nul et que par suite $N_{1}$ est nul. Ceci veut dire aussi que dans (16) on ne doit tenir compte que des valeurs de $y$ comprises entre $y^{\prime}$ et 1 par suite $V_{2}$ se réduit à :

$$
\begin{aligned}
N_{2}^{\prime \prime}=\int_{R+z}^{R+z^{\prime}} r \rho(r)\left[\frac{1}{\sqrt{r^{2}-(R+z)^{2} \sin ^{2} \varphi}}\right. \\
\left.-\frac{1}{\sqrt{r^{2}-R^{2} \sin ^{2} \varphi}}\right] \mathrm{d} r
\end{aligned}
$$

cette quantité sera toujours très petite comme on le reconnaît à l'aide du développement toujours très convergent :

$$
\begin{aligned}
N_{2}^{\prime}=\int_{R+z}^{R+z^{\prime}} & \frac{r p(r)}{\sqrt{r^{2}-R^{2} \sin ^{2} \varphi^{2}}}\left[\frac{1}{2} \frac{2 R z+z^{2}}{r^{2}-R^{2} \sin ^{2} \cdot} \sin ^{2} ?\right. \\
& \left.+\frac{1.3}{2.4}\left(\frac{2 R z+z^{2}}{r^{2}-R^{2} \sin ^{2} \varphi}\right)^{2} \sin ^{4} \varphi+\ldots\right] \mathrm{d} r .
\end{aligned}
$$

Nous négligerons cette quantité comme Cabannes et Dufay l'ont fait, implicitement d'ailleurs.

Il reste alors :

$\operatorname{Lg} \frac{I}{I^{\prime}}=-\left(K-K^{\prime}\right) N_{0}+\operatorname{Lg} \frac{\int_{0}^{\prime} \mathbf{e}^{-d\left(m_{0}-1\right) y} \mathrm{~d} y}{\int_{0}^{\prime} \mathbf{e}^{-d^{\prime}\left(m_{0}-1\right) y d} d y}+\mathrm{C}^{\mathrm{te}}$.

En appelant $\frac{I_{1}}{I_{1}}$ le rapport qu'on aurait mesuré en l'absence d'ozone on a

$$
\operatorname{Lg} \frac{I}{I^{\prime}}=\operatorname{Lg} \frac{I_{1}}{I^{\prime}{ }_{1}}-\left(K-K^{\prime}\right) N_{0},
$$

ce qui est l'équation de Cabannes et Dufay.

Passons au cas général où on ne peut plus négliger $N_{1}$ et $N_{2}$. On obtient :

$$
\begin{aligned}
& \left(K-K^{\prime}\right) N_{0}=\left(K-K^{\prime}\right) \int_{R}^{\infty} \frac{r \wp(r) \mathrm{d} r}{\sqrt{r^{2}-R^{2} \sin ^{2} \varphi}} \\
& =\operatorname{Lg} \frac{\int_{0}^{\prime} \mathrm{e}^{-h\left(N_{1}+N_{2}\right)} \mathrm{e}^{-d\left(m_{0}-1\right) y} \mathrm{~d} y}{\int_{0}^{\prime} \mathrm{e}^{-h^{\prime}\left(N_{1}+N_{2}\right)} \mathrm{e}^{-d^{\prime}\left(m_{0}-1\right) y \mathrm{~d} y}-\operatorname{Lg} \frac{I}{I^{\prime}}+\mathrm{C}^{\mathrm{te}}}
\end{aligned}
$$

Si nous avons pour $\rho(r)$ une première approximation on pourra calculer $N_{1}$ et $N_{2}$ et par suite le deuxième membre de 17 , sera connu. L'équation proposée a alors la même forme que l'équation (1) que nous savons maintenant résoudre. Sa résolution fait connaître une nouvelle approximation de $\rho(r)$ avec laquelle on reprendra le calcul et ainsi de suite jusqu'à ce qu'on juge suffisante l'approximation obtenue.

Il ne sera pas en général avantageux de prendre comme première approximation $N_{1}=N_{2}=0$, car il semble bien, d'après les mesures de Dobson en particulier, que cette approximation soit assez éloignée de la réalité. Il sera préférable d'utiliser une formule plus ou moins empirique, par exemple une courbe de Gauss

$$
p(r)=\frac{1}{\sigma \sqrt{2 \pi}} \mathbf{e}^{-\frac{(r-b)^{2}}{2 \sigma^{2}}}
$$

avec une valeur de $b$ voisine de $R+50 \mathrm{~km}$ et $\sigma$

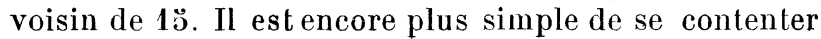
d'une couche mince vers $50 \mathrm{kms}$ et nous montrerons que cette approximation conduit déjà à des constatations intéressantes. La deuxième approximation sera très facile à calculer mais il faut bien avouer que si 
l'on juge insuffisant le résultat obtenu, leṣ calculs ultérieurs seront pénibles.

10. Cas d'une couche mince. - Dans ce cas le problème se simplifie

Pour $z<h: \quad N_{2}=0$

et $\quad N_{1}=\varepsilon D\left[\frac{1}{\sqrt{D^{2}-(R+z)^{2} \sin ^{2} \psi}}-\frac{1}{\sqrt{D^{2}-R^{2} \sin ^{2} \varphi}}\right]$.

Pour $z>h$ :

$$
N_{1}=0 \quad \text { et } \quad N_{2}=\varepsilon\left[1-\frac{1}{\sqrt{U^{2}-R^{2} \sin ^{2} p}}\right] .
$$

On a d'ailleurs

$$
\begin{aligned}
N_{1}=\frac{\varepsilon D}{\sqrt{D^{2}-R^{2} \sin ^{2} \varphi}}\left[\frac{1}{2} \frac{2 z R+z^{2}}{D^{2}-R^{2} \sin ^{2} \varphi} \sin ^{2} \varphi\right. \\
\left.\quad+\frac{1,3}{2,1}\left(\frac{2 z R+z^{2}}{D^{2}-R^{2} \sin ^{2} \varphi}\right)^{2} \sin ^{4} \varphi+\ldots\right]
\end{aligned}
$$

en se limitant au terme principal et en passant à la variable ‘ définie par (4).

$$
N_{1}=\frac{\varepsilon z}{R} \sec \psi \operatorname{tg}^{2} \psi \quad \text { pour } \quad z<h
$$

et

$$
N_{2}=\varepsilon(1-\sec \psi) \quad \text { pour } \quad z>h .
$$

soit encore

$$
y_{0}=\mathbf{e}^{-A h} .
$$

L'équation (17) devient alors

$$
\operatorname{Lg} \frac{\boldsymbol{I}}{\boldsymbol{I}^{\prime}}=\mathrm{C}^{\mathrm{te}}-\left(\boldsymbol{K}-\boldsymbol{K}^{\prime}\right) \boldsymbol{\Lambda}_{0}^{\tau}+\boldsymbol{X}(\psi)
$$

où l'on $\mathbf{a}$

$$
\mathrm{Lg} \frac{\mathbf{e}^{-K N_{2}} \int_{0}^{y_{0}} \mathbf{e}^{-d\left(m_{0}-1\right) y} \mathrm{~d} y+\int_{y_{0}}^{1} \mathbf{e}^{-K N_{1}} \mathbf{e}^{-l\left(m_{0}-1\right.} y \mathrm{~d} y}{\mathbf{e}^{-K^{\prime} N_{z}} \int_{0}^{y_{0}} \mathbf{e}^{-d\left(m_{0}-1\right) y} \mathrm{~d} y+\int_{y_{0}}^{\prime} \mathbf{e}^{-K^{\prime} \nu_{2}} \mathbf{e}^{-d^{\prime}\left(m_{0}-1\right) y} \mathrm{~d} y}
$$

et

$$
N_{0}=\varepsilon \sec \psi \text {. }
$$

On a :

$$
\int_{0}^{y_{0}} \mathbf{e}^{-d\left(m_{0}-1\right) y} \mathrm{~d} y=\frac{1-\mathbf{e}^{-d\left(m_{0}-1\right) y_{0}}}{d\left(\boldsymbol{m}_{0}-1\right)} .
$$

Posons en outre :

$$
\begin{aligned}
d\left(m_{0}-1\right) y & =u & & d^{\prime}\left(m_{0}-1\right) y=u^{\prime} \\
d\left(m_{0}-1\right) y_{0} & =u_{0} & & d^{\prime}\left(m_{0}-1\right) y_{0}=u_{0}^{\prime} \\
d\left(m_{0}-1\right) & =u_{1} & & d^{\prime}\left(m_{0}-1\right)=u_{1}^{\prime} \\
\frac{K^{\prime}}{A R} \sec \psi \operatorname{tg}^{2} \psi & =\alpha & \frac{K^{\prime} \varepsilon}{A R} \sec \psi \operatorname{tg}^{2} \psi & =\alpha^{\prime}
\end{aligned}
$$

il vient après quelques réductions simples

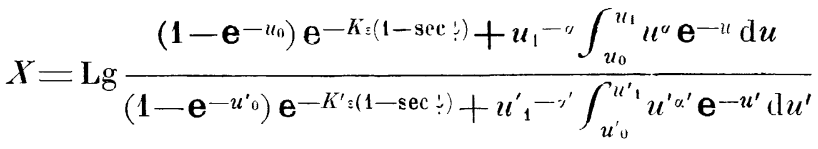

$$
\begin{aligned}
& +\operatorname{Lg} \frac{d^{\prime}}{d} .
\end{aligned}
$$

Nous ne nous occuperons plus du terme $\operatorname{Lg} \frac{d^{\prime}}{d}$ que nous faisons entrer dans la constante de l'équation (18).

On voit d'après l'expression de $X^{\prime}$ que ce terme est petit pour les pelites valeurs de $\psi$ puis qu'il croît ensuite. Le maximum qu'il puisse atteindre pour $h$ petit et $\downarrow$ grand est

$$
-\left(K-K^{\prime}\right) \varepsilon(1-\sec \psi)
$$

Dans ce cas, à l'horizon, le rapport des intensités $\frac{I}{l^{\prime}}$ est le même qu'au zénith. Il passe par un minimum entre ces deux points.

Nous avons calculé la fonction $X^{\prime}$ pour des valeurs de $h$ de 30 et $50 \mathrm{kms}$ avec $\varepsilon=1,3 \mathrm{~cm}$. Les radiations $\lambda$ et $\lambda^{\prime}$ ayant les longueurs d'ondes 3110 et $3265 \AA$. On a pris $A=\mathbf{0 , 1 4}$ et les valeurs de $K, K^{\prime}, d, d^{\prime}$ utilisées par Chalonge dans son mémoire. Enfin $m_{0}$ est extrait d'une table établie par Bemporad $\left({ }^{1}\right)$.

Le calcul ne présente pas de grandes difficultés. Seules les intégrales

$$
\int_{u_{0}}^{u_{1}} u^{\prime} \mathbf{e}^{-u} \mathrm{~d} u \quad \text { et } \quad \int_{u_{0}^{\prime}}^{u_{1}^{\prime}} u^{\prime u^{\prime}} \boldsymbol{\epsilon}^{-u^{\prime}} \mathrm{d} u^{\prime}
$$

demandent un peu d'attention.

On a

$y_{0} \alpha\left(\boldsymbol{\epsilon}-u_{1}-\mathbf{e}^{-u_{1}}\right)<u_{1}-u \int_{u_{0}}^{u_{1}} u^{\alpha} \mathbf{e}^{-u} \mathrm{~d} u<\mathbf{e}^{-u_{0}}-\mathbf{e}^{-u_{1}}$

et aussi

$$
\begin{aligned}
& u_{1}-\int_{u_{0}}^{u_{1}} u^{\alpha} \mathbf{e}^{-u} \mathrm{~d} u \\
& =u_{1}-\left[\left[\Gamma(1+x)-\int_{0}^{u_{0}} u^{\prime \prime} \mathrm{e}^{-u} \mathrm{~d} u-\int_{1}^{\infty} u^{\prime \prime} \mathbf{e}^{-u} \mathrm{~d} u\right] .\right.
\end{aligned}
$$

Si les inégalités $\left(2_{11}\right)$ domnent des limiles trop écartées, on calcule les intégrales à l'aide de (21). Les intégrales qui figurent dans celle formule, au second membre, sont généralement très petites et il cst en tout cas facile d'en obtenir des valeurs approchées.

La figure 2 montre comment varie le rapport $\frac{I}{I^{\prime}}$ avec la dislance zénithale $\varphi$. Les abcisses ont été prises proportionnelles à sec $\psi($ pour $h=50 \mathrm{kms}$ ).

Les courbes $\Gamma_{1}$ et $\Gamma_{2}$ sont celles données par le terme en sec $\nu$, (elles sont peu différentes de l'approximation de Cabannes et Dufay) pour les altitudes d'une couche

(') Handbuch der Astrophysik II, 1, p. 26 ?. 
mince de 50 et $30 \mathrm{kms}$ respectivement. Les courbes $\Gamma_{1}^{\prime}$ et $\Gamma_{2}{ }_{2}$ résultent de notre calcul.

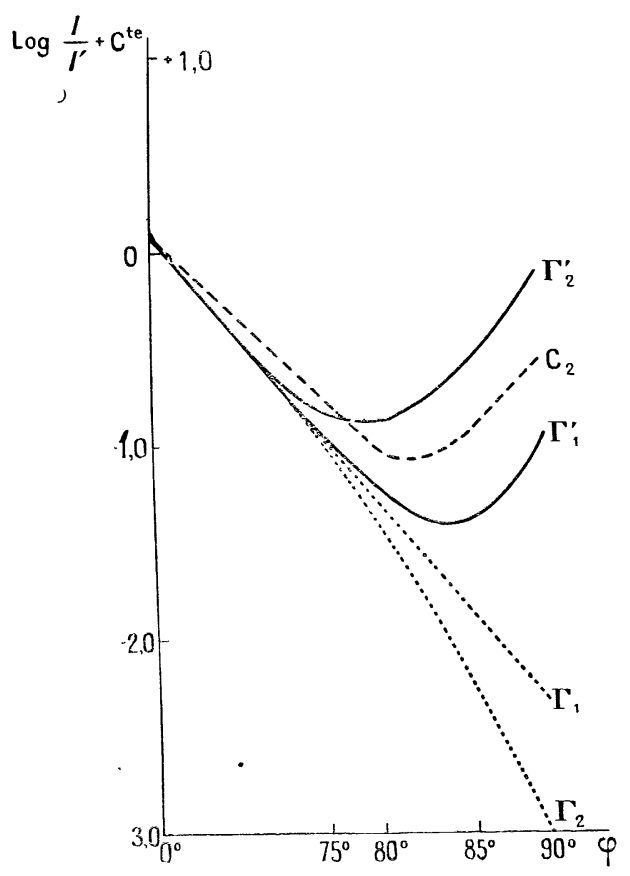

Fig. 2.

La courbe $\mathrm{C}_{2}$ est l'une des courbes expérimentales de Dobson publiées par Chalonge. Elle correspond à une épaisseur d'ozone de $0,266 \mathrm{~cm}$ seulement, ce qui explique que sa tangente à l'origine ne coïncide pas avec celles de nos courbes. Compte tenu de ce fait, il semble qu'elle puisse se représenter par une couche mince dont l'altitude serait de l'ordre de 40 à $50 \mathrm{~km}$. $\mathrm{Si}$ on arrivait à une représentation parfaite, on ne pourrait pas dire toutefois que l'ozone est certainement réparti en une couche mince, car il paraît bien évident à priori que des répartitions plus compliquées conduiraient au même résultat. Il faut encore remarquer que l'altitude de la couche mince n'a plus ici de sens physique très net, ce n'est plus l'altitude moyenne de la distribution d'ozone au sens strict du mot. Toutefois le résultat numérique qu'on vient d'obtenir montre qu'elle ne doit pas en différer beaucoup.

Le point le plus important à retenir est que la diffusion aux trés hautes allitudes joue un rôle capital pour écarter les courbes expérimentales des courbes obtenues dans l'hypothèse simple de Cabanmes et Dufay. La différence d'absorption subie entre un rayon rencontrant la verticale à une très grande altitude et un autre à très faible altitude est formidable et montre que cette constatation n'est pas extraordinaire. Dans ces recherches sur ce sujet, Chalonge avait arbitraire. ment limité à $30 \mathrm{~km}$ l'altitude des couches diffusantes, pour rendre compte des observations, il avait dù imaginer deux couches l'une en dessous, l'autre en dessus de cette limite. Ceci lui permettait d'affirmer qu'il y avait une proportion très notable d'ozone en dessous de $30 \mathrm{~km}$. D'après la théorie que nous venons d'établir, il ne semble pas que les observations permeltent de confirmer ce résultat.

D'après l'équation (20), on voit que la fonction $m_{0}$ joue un rôle très important dans le calcul, d'autant plus important qu'elle n'est pas multipliée par un nombre petit $\left(d-d^{\prime}\right)$ comme dans la méthode de Fabry et Buisson. Ce que nous avons déjà dit au sujet de cette fonction prend ici une importance capitale. $I l$ est indispensable de la déterminer pour chaque série d'observation. Il serait également souhaitable de déterminer en chaque point de l'atmosphère, la densité de l'air et ceci n'est pas absolument impossible. Enfin il faudrait préciser l'effet de la diffusion secondaire ou en général des diffusions d'ordre élevé. Dans l'état actuel de la question, tout ce qu'on peut dire est que les observations effectuées par la méthode de Cabannes et Dufay peuvent être interprétées dans l'hypothèse d'une couche mince qui, d'après les mesures de Dobson, serait à une altitude de $40 \mathrm{~km}$ environ.

11. Conclusions. - La méthode de Fabry et Buisson conduit à des résultats facilement interprétables et il n'en est pas de mème pour la méthode de Cabannes et Dufay. Ces deux méthodes ne peuvent pas en pratique déterminer avec une approximation suffisante la répartition de l'ozone en fonction de l'altitude. Il semble bien probable que ce problème soit insoluble d'une manière satisfaisante avec des observations faites au sol seulement. Au contraire, si on pouvait combiner les résultats obtenus par la méthode de Fabry et Buisson avec des dosages chimiques effectués en ballon jusqu'à des altitudes de l'ordre de $15 \mathrm{~km}$ environ, on pourrait considérer le problème comme résolu. Techniquement, rien ne s'oppose à la réalisation d'un tel projet. 